\title{
Application of GPR Method for Detection of Loose Zones in Flood Levee
}

\author{
Tomisław Gołębiowski ${ }^{1, *}$, Tomasz Małysa ${ }^{2}$ \\ ${ }^{1}$ Cracow Uniwersity of Technology, Faculty of Environmental Engineering, Institute of Geotechnics, \\ Department of Geodesy, Geophysics and Engineering Geology, Warszawska st. 24, Cracow, Poland \\ ${ }^{2}$ Firm: MET-GEO, Styczniowa st. 37, Trzebinia, Poland
}

\begin{abstract}
In the paper the results of non-invasive georadar (GPR) surveys carried out for detection of loose zones located in the flood levee was presented. Terrain measurements were performed on the Vistula river flood levee in the village of Wawrzeńczyce near Cracow. In the investigation site, during the flood in 2010, leakages of levee were observed, so detection of inner water filtration paths was an important matter taking into account the stability of the levee during the next flood. GPR surveys had reconnaissance character, so they were carried out with the use of short-offset reflection profiling (SORP) technique and radargrams were subjected to standard signal processing. The results of surveys allowed to outline main loose zone in the levee which were the reason of leakages in 2010. Additionally gravel interbeddings in sand were detected which had an important influence, due to higher porosity of such zones, to water filtration inside of the levee. In the paper three solutions which allow to increase quality and resolution of radargrams were presented, i.e. changeable-polarisation surveys, advanced signal processing and DHA procedure.
\end{abstract}

\section{Introduction}

Detection of inner damages in hydrotechnical constructions is very important matter because such damages (e.g. loose zones in flood levees or fractures in concrete dams etc.) may lead to leakage of those construction and in the extreme case (e.g. during the flood) to the destruction of levees and dams. If damages did not appear on the surface of hydrotechnical construction it's impossible to know of its existence. Therefore it is very important to find the methods for examination of inner condition of hydrotechnical construction. Such examination may be carry out on the basis of the boreholes and analysis of material obtained from them or mounting in the constructions different sensors. Both methods weaken the construction, are invasive and expensive and, which is the most important, delivers only punctual information. Solution of described problems might be the application of non-invasive geophysical methods which delivered continuous information between boreholes and sensors. Method presented in the paper may be applied for examination of different hydrotechnical constructions but authors focused only on noninvasive examination of flood levees.

\footnotetext{
*Corresponding author: goleb@,wis.pk.edu.pl, tomaszmalysa1984@,02.pl
} 
There is may geophysical methods which may be applied for examination of flood levees; generally electrical and electromagnetic methods were applied for this purpose, i.e. Electrical Resistivity Tomography (ERT), Electromagnetic Profiling (EMP), Ground Penetrating Radar (GPR) and occasionally Capacitively Coupled Resistivity method [1 4]; increasingly Multichannel Analysis of Surface Waves (i.e. MASW technique) is applied for examination of flood levees. Very seldom microgravimetric surveys were applied for location of loose zones in the levees. In the paper authors focused only on the GPR method.

\section{Investigation site}

GPR surveys were carried out on the flood levee of the Vistula river in the village of Wawrzeńczyce near Cracow (Fig. 1). The levee in the investigation site were built before World War II and renovated in years 1970-72. During the flood in 2010, flood gate was partly destroyed and in the body of the levee leakages were observed (Fig. 1 - places with marks no. I, II, III, IV and V). After the flood, the levee in place no. III (Fig. 1) and flood gate were reconstructed. Other parts of the levee where leakages were observed during the flood were only protected with bags with sand (Fig. 1) and no renovation was carried out. Therefore the aim of GPR surveys was detection of loose zones existed in the levee which were water filtration paths and during the next flood may threaten the stability of the levee.

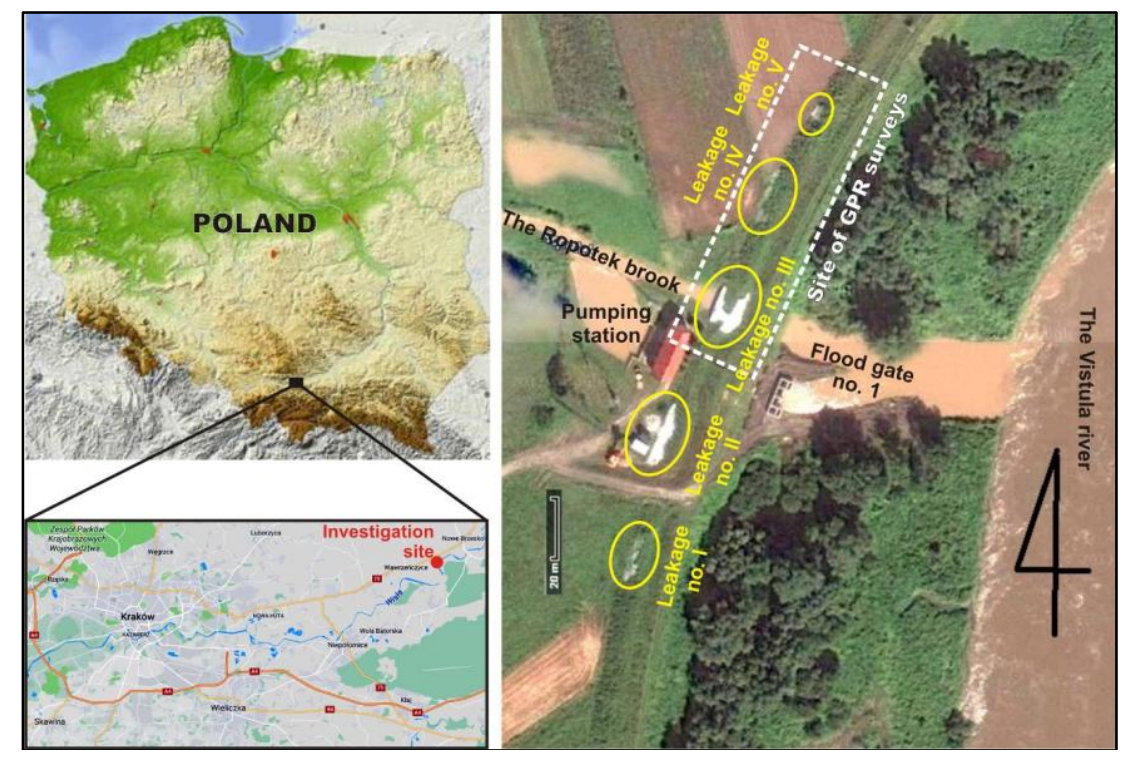

Fig. 1. GPR investigation site on the flood levee of the Vistula river in the village of Wawrzeńczyce near Cracow (base maps: www.google.pl/maps).

Reconnaissance geophysical (i.e. ERT and GPR - $100 \mathrm{MHz}$ ) surveys and geotechnical (i.e. DLP) investigations were carried in this site in 2014 and the results were presented in scientific conference in Korbielów (Poland), in 2015. High resolution GPR $-300 \mathrm{MHz}$ surveys were performed in 2016 and selected results were presented in scientific conference in Korbielów, in 2017. In this paper authors would show and discuss all results of GPR $300 \mathrm{MHz}$ surveys and present additional solutions which allow to increase quality and resolution of radargrams recorded on flood levee, i.e. changeable-polarisation surveys, advanced signal processing and DHA procedure. GPR surveys, in 2D mode, were carried 
out on the top and on the shelfs of the levee (Fig. 2). On the basis of borehole information (Fig. 3) inner construction of the levee in the investigation site was known (Fig. 3). Geotechnical investigations delivered information that body of the levee is built mainly of sand which is compacted and semi-compacted but in several places of the levee interbeddings and accumulations of gravel occurred.

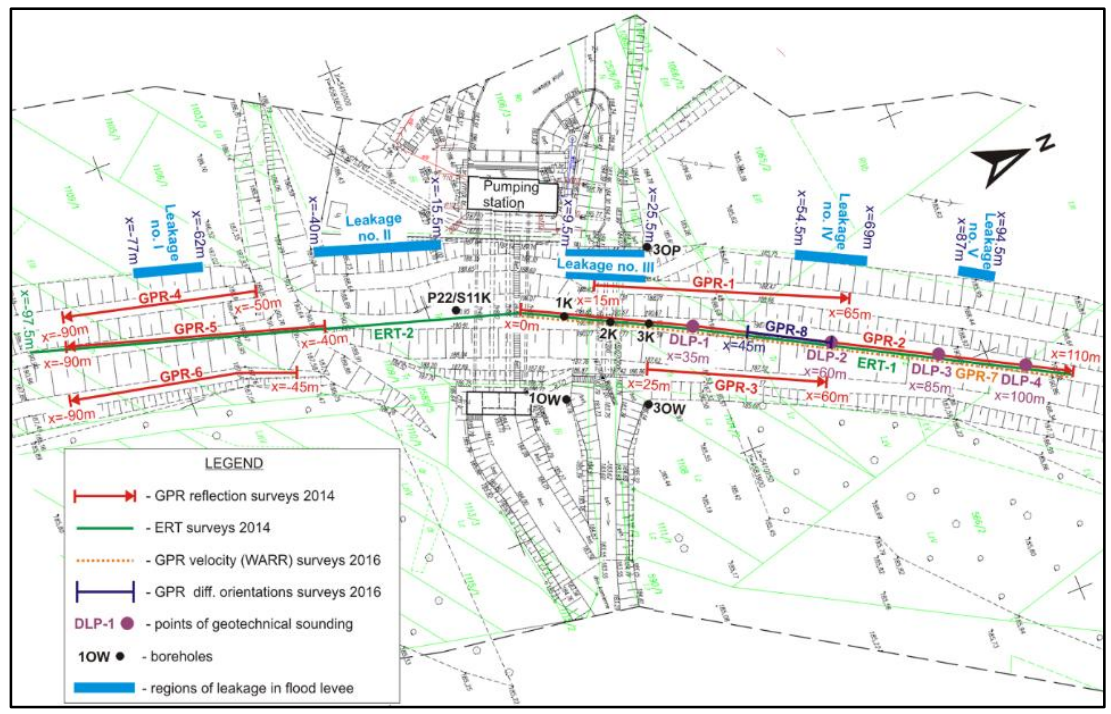

Fig. 2. Project of geophysical and geotechnical investigations (base map: [5]).

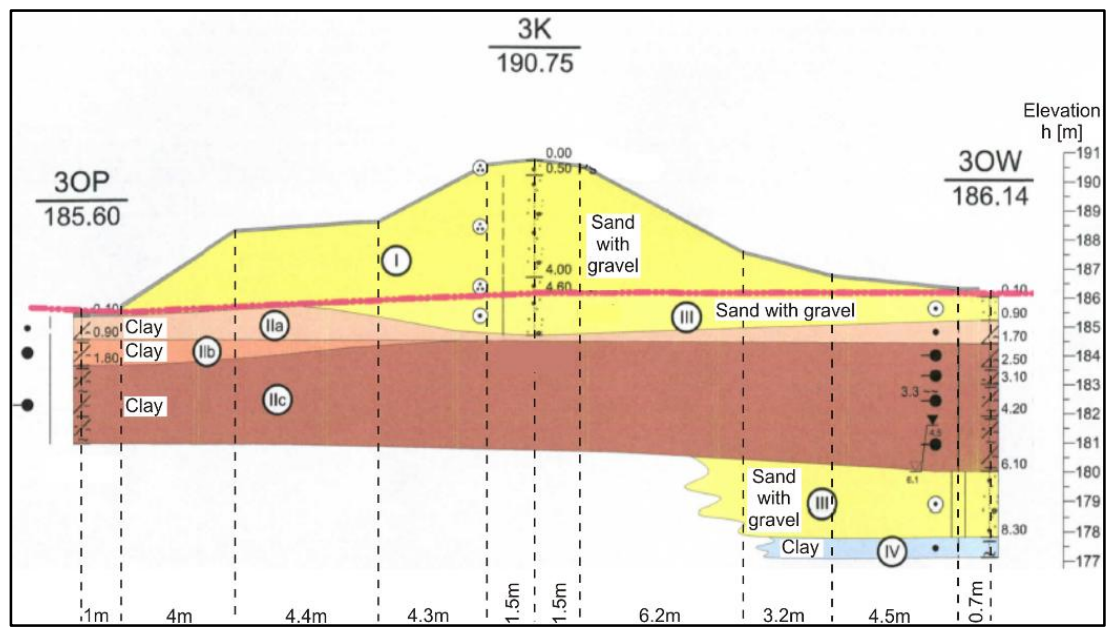

Fig. 3. Geological information from the investigation site [5].

\section{Fundaments of GPR method}

In the GPR method many different measurement techniques are known, but for reconnaissance surveys the short-offset (occasionally zero-offset) reflection profiling (SORP) technique is used. In SORP technique the transmitter (Tx) and receiver (Rx) antennae move along the profile (Fig. 4A), where the transmitter antenna emits electromagnetic signals at every specified distance interval $(\Delta \mathrm{x})$. The signals propagate in the form of an electromagnetic (e-m) wave within the geological medium (or examined 
construction), reflecting from anomalous bodies and geological boundaries (Fig. 4A). The reflected waves are recorded by the receiver antenna and displayed on the computer during the measurement (Fig. 4A) in the form of a radargram (Fig. 4B). The horizontal axis on the radargram is recorded on the length scale, whereas the vertical axis is recorded on the time scale; during the processing of measurement data, the time axis is converted into the depth axis, taking into consideration the information about the velocity of the e-m wave within the studied medium. Depending of type of analysed problem, antennae with different constructions and different frequencies are used. SORP technique is fast, cheap and measurements may be performed by only one person (Fig.4A) but disadvantage of this technique is fact that only qualitative interpretation may be performed. More information concerning SORP technique might be found, between others, in book by [6].

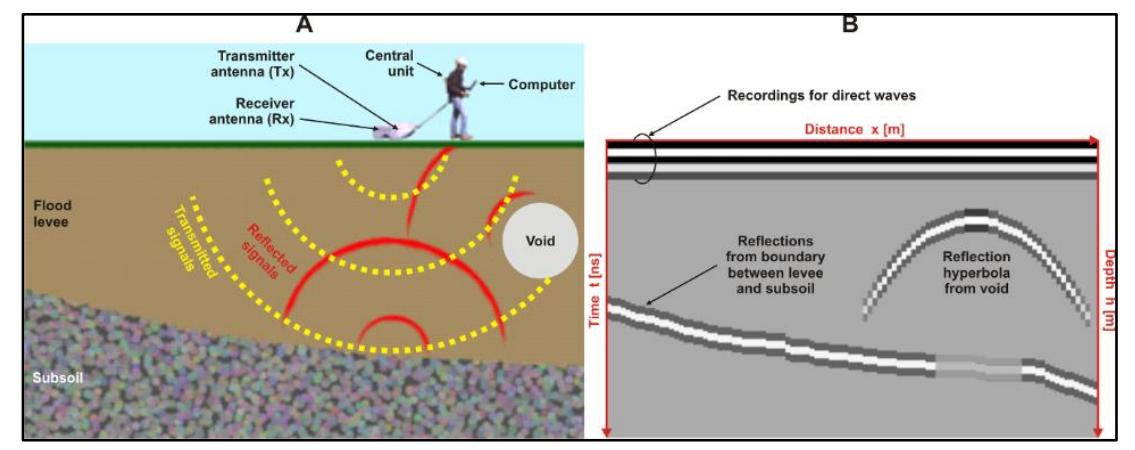

Fig. 4. A) GPR SORP technique; B) Recorded radargram ([7] - changed).

The contrast of values of relative electrical permittivity $\varepsilon_{r}$ (Table 1 ), between the anomalous body (e.g. void or loose zone in the levee) and the surrounding medium (e.g. compacted part of the levee) or between two geological materials (Fig. 4A) decides upon the value of the reflection coefficient $R$ [-], and in consequence upon the amplitude of the reflections recorded on the radargram (Fig. 4B). For SORP technique and for media analysed in the paper reflection coefficient may be simplified to formula (1). Relative electrical permittivity $\varepsilon_{r}$ defines also value of velocity of e-m wave in the examined medium (Table 1), according to simplified formula (2). The value of the electrical conductivity $\sigma$ (Table 1 ) decides upon the attenuation $\alpha$ of the e-m wave (Table 1); for media analysed in the paper (Fig. 3), which may be treated as lossless media, attenuation might be defined by simplified formula (3).

$$
R=\frac{\sqrt{\varepsilon_{r_{-} \text {medium }}}-\sqrt{\varepsilon_{r_{-} \text {anomaly }}}}{\sqrt{\varepsilon_{r_{-} \text {medium }}}+\sqrt{\varepsilon_{r_{-} \text {anomaly }}}} \quad v=\frac{30}{\sqrt{\varepsilon_{r}}} \quad \alpha \approx 1.7 \cdot 10^{3} \frac{\sigma}{\sqrt{\varepsilon_{r}}}
$$

In SORP technique quality of radargram, so in consequence possibility of proper interpretation, may be increased in four ways, i.e.:

1) by carrying out the surveys with changeable orientation (changeable polarisation) of antennae (Fig. 5),

2) by application of procedure of Diffraction Hyperbola Analysis (DHA) which allows to estimate velocity of examined medium,

3) by application of advanced signal processing and digital images analysis,

4) by application of antennae with different constructions and modern electronic solutions. 
Table 1. Properties of media analysed in the paper [7].

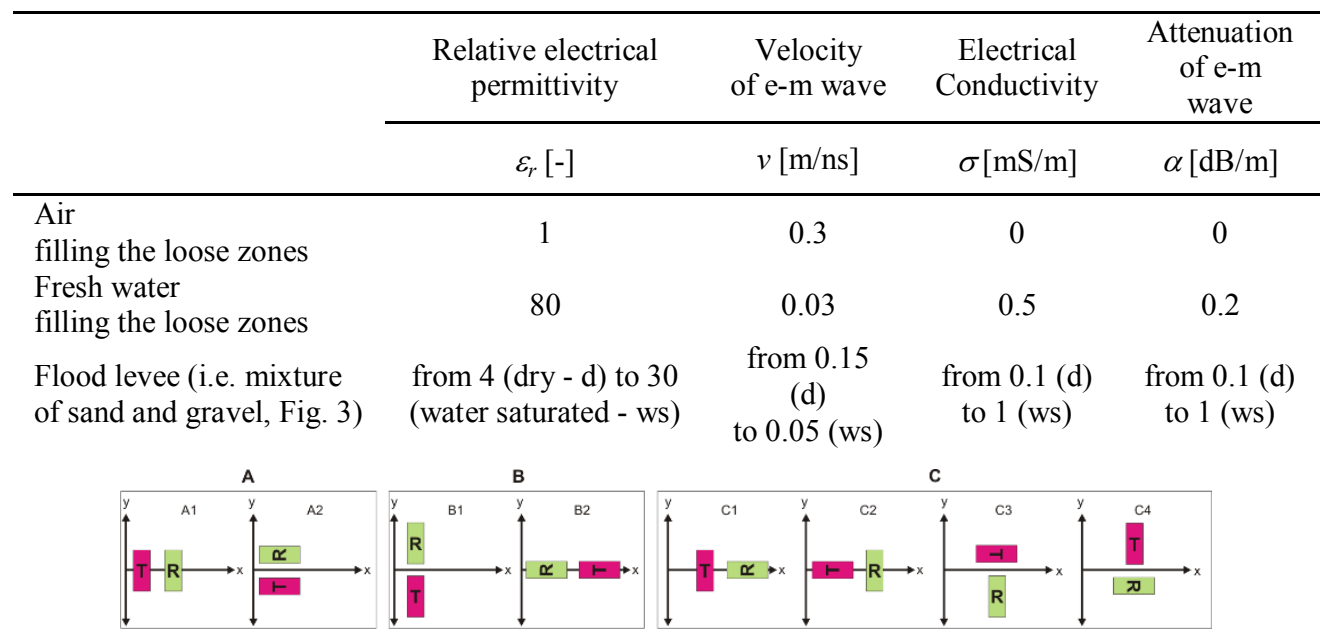

Fig. 5. Different orientations of GPR antennae: A) "co-pole" orientation; B) "end-fire" orientation; C) "cross-pole" orientation.

Results of SORP surveys carried out in 2014 with using of standard orientation of antennae (Fig. 5A - option A1) was shown in Fig. 6 and Fig. 7. Terrain surveys were carried out with the use of ZOND georadar (produced by Radar Systems Inc. www.radsys.lv) and $300 \mathrm{MHz}$ antenna which max. depth penetration is c.a. $10 \mathrm{~m}$ and mean resolution is c.a. $0.2 \mathrm{~m}$ was applied for surveys. Traces were recorded with constant distance interval $\Delta \mathrm{x}=0.05 \mathrm{~m}$. For time-depth conversion mean velocity equal $0.1 \mathrm{~m} / \mathrm{ns}$ was assumed on the basis of application of Diffraction Hyperbola Analysis procedure (Fig. 6C). All radargrams was subjected to standard signal processing in ReflexW program (www.sandmeiergeo.de). In Fig. 6A weathered and loose zone (anomaly no. 1) is easily noticed; leakage in place no. IV was an effect of presence of loose zone located by the basement of the levee (deeper part of anomaly no. 1); inner boundary (anomaly no. 2) is an effect generated by presence of gravel in sand. In Fig. 6B high-amplitude anomaly no. 3 generated by presence of loose zone was recorded; this anomaly was the reason of leakage in place no. III; linear anomaly no. 4 was generated by gravel interbedding located in sand; anomaly no. 5 , generated by presence of loose zone, was the reason of leakage of the levee in place no. V. In Fig. 6C weathered and loose zone was recorded by the surface (anomaly no. 6); interesting anomaly no. 7 was recorded in subsoil of the levee and it may indicate the location of highly loose zone or even void which was the reason of leakage in place no. IV. In Fig. 7A two anomalies, i.e. no. 8 (weathered and loose material) and no. 9 (loose zone) were distinguished; leakage observed in 2010 in place no. I was caused by presence of anomalies no. 8 and 9. In Fig. 7B loose and weathered material is clearly visible (anomaly no. 11); gravel interbedding in sand is presented in Fig. 7B as anomaly no. 10. In Fig. 7C one large anomaly no. 12 (i.e. weathered and loose zone) was detected by the surface. Generally body of the levee in the investigation site is disintegrated. Additionally gravel interbeddings located in sand characterise themselves by the higher porosity and water permeability. Mentioned facts cause that during the next flood leakages and seepages will appear and stability of the levee may be threaten.

Unfortunately in many cases standard orientation of GPR antennae delivers worst information than application of non-standard orientation. In Fig. 8 results of surveys carried out in former plant was presented. Remains of tank pad, located underground is only 
fragmentary visible in Fig. 8A ("co-pole" orientation) and trenched area where leakage of creosote from tank took a placed is visible.
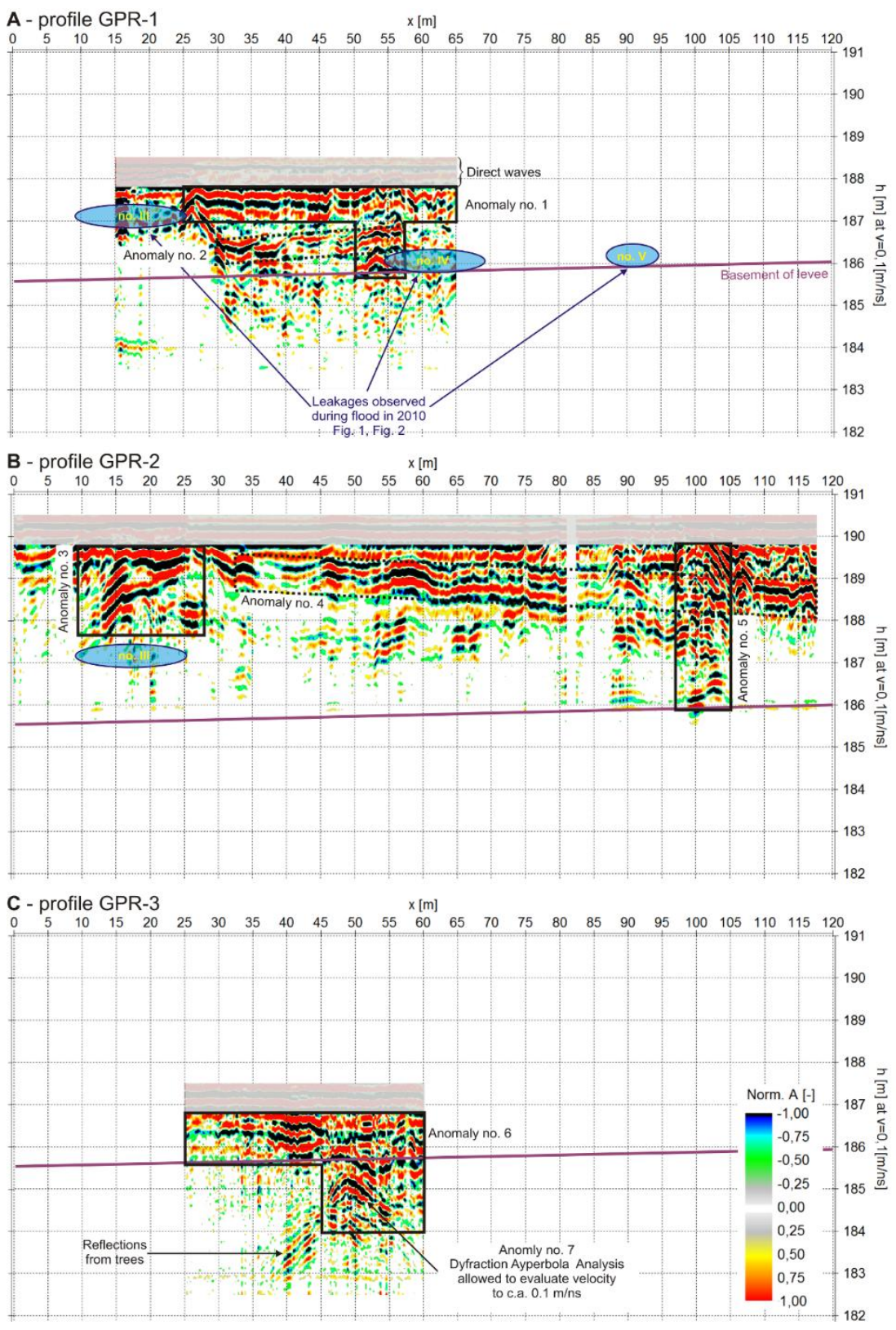

Fig. 6. Radargrams recorded on the levee for profiles: GPR-1 (A), GPR-2 (B), GPR-3 (C) 

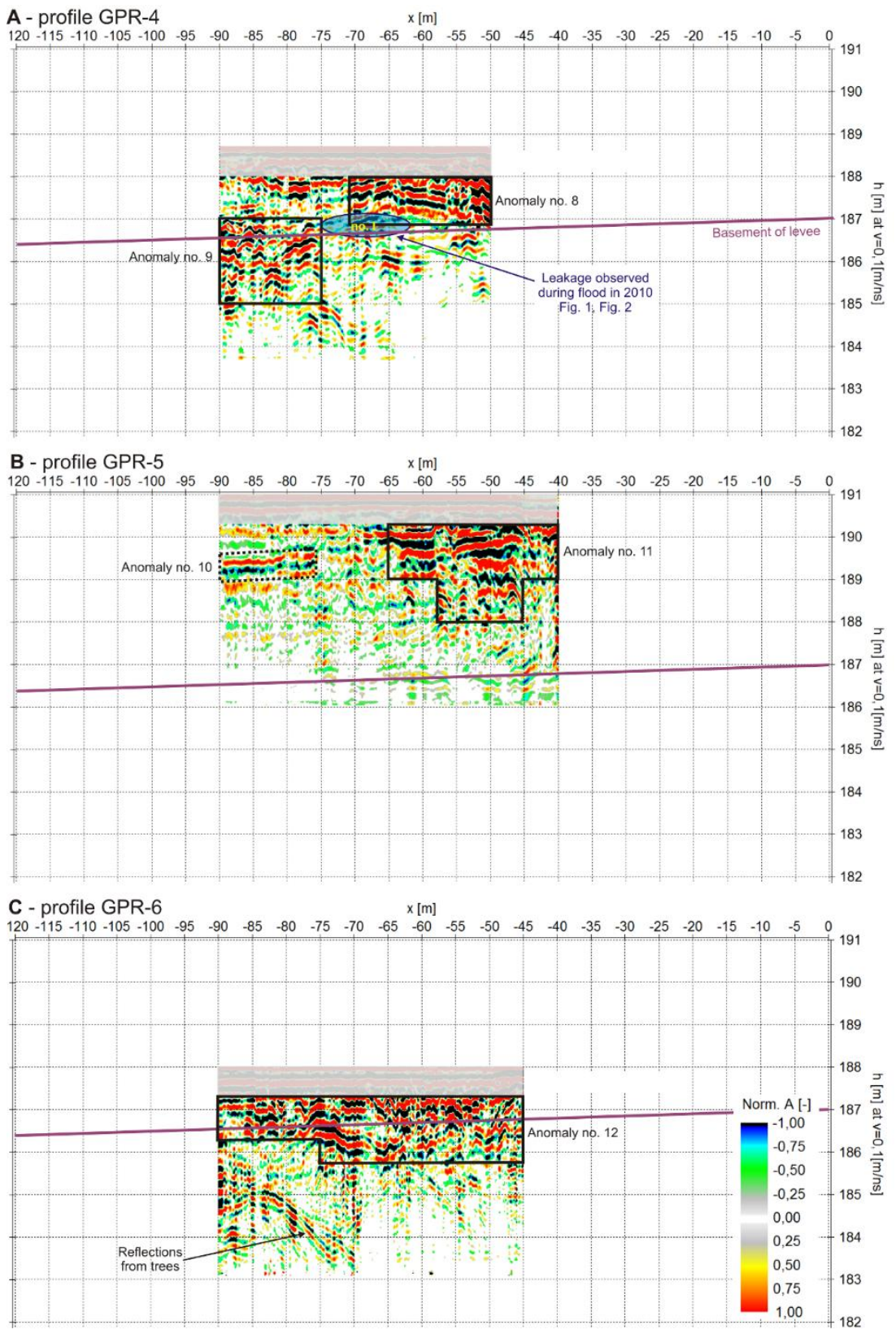

Fig. 7. Radargrams recorded on the levee for profiles: GPR-4 (A), GPR-5 (B), GPR-6 (C)

In Fig. 8B ("cross-pole" orientation) shape of tank pad is much better visible than in Fig. 8A, trench (i.e. brake of continuity of tank pad) is also visible; additionally in Fig. 8B rebars in tank pad may be distinguished. Application of non-standard orientation of antennae (Fig. 8B) allowed also to depict creosote-filled vault which was impossible in Fig. 8A. It is highly probable that application of different orientations of antennae during the surveys $[8,9,10]$ on the levee in Wawrzeńczyce site delivered more detailed information. It should be emphasized that the vast majority of the research conducted on the flood embankments is carried out using the SORP technique with standard orientation of 
GPR antennae. Not all contractors have bistatic antennas and can not perform tests using different dipole orientations $[11,12]$.

A

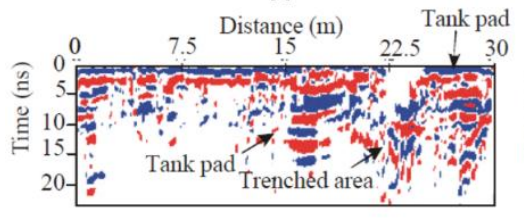

B

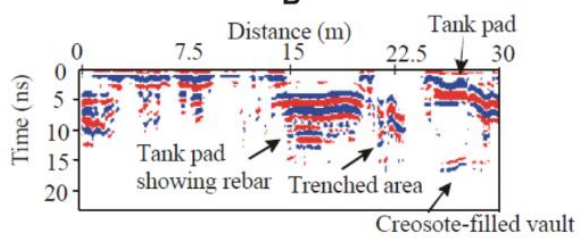

Fig. 8. GPR SORP surveys with the use of standard "co-pole" (A) and non-standard "cross-pole" (B) orientations of antennae [8].

Additional information needed for quantitative interpretation of the results of SORP surveys may deliver application of DHA procedure. This procedure allows to analyse velocity of e-m weave in places of radargram where hyperbolae appeared (like it was shown in Fig. 6C - anomaly no. 7). Information about velocity distribution (Fig. 9A) is very important because it allows to perform proper time-depth conversion of radargram and additionally allows to construct a map of changes of velocity in the examined medium (Fig. 9B); on the basis of such map, distribution of porosity and water saturation of the loose zones may be solved on the basis of formulae (2), Topp formula (4), BHS formula (5) and CRIM formula (6); in consequence quantitative interpretation is possible. Detailed description of velocity analysis and transformation of velocity map into porosity or water saturation maps may be found in works Gołębiowski $(2010,2012)$. In order to demonstrate DHA procedure, in Fig. 9A shown example radargram with hypothetical diffraction hyperbolae, because real unfortunately was not recorded; in Fig. 9B velocity map solved on the basis of DHA procedure was presented. Unfortunately during SORP surveys of flood levees very seldom few or dozen and so diffraction hyperbolae (like in Fig. 9A) are recorded (see Figs. 6, 7, 8); additionally for construction of proper velocity map, diffraction hyperbolae have to be distributed uniformly on the radargram (Fig. 9A).

$$
\begin{gathered}
\theta_{w}=4.3 \cdot 10^{-6} \cdot \varepsilon_{r}^{3}-5.5 \cdot 10^{-4} \cdot \varepsilon_{r}^{2}+2.92 \cdot 10^{-2} \cdot \varepsilon_{r}-5.3 \cdot 10^{-2} \\
v=\frac{c}{\sqrt{\left[\theta_{w} \cdot \varepsilon_{r_{-} w}^{\alpha}+(1-n) \cdot \varepsilon_{r_{-} g}^{\alpha}+\left(n-\theta_{w}\right) \cdot \varepsilon_{r_{-} a}^{\alpha}\right]^{\frac{1}{\alpha}}}} \\
n=\left[\frac{\left(\frac{c}{v}\right)^{2}-\varepsilon_{r_{-} w}}{\varepsilon_{r_{-} w}-\varepsilon_{r_{-} g}}\right] \cdot\left[\frac{\varepsilon_{r_{-} w}}{\left(\frac{c}{v}\right)^{2}}\right]^{\beta}
\end{gathered}
$$

where $\theta_{w}$ is volumetric water content, $n$ is porosity, $\varepsilon_{r_{-} w} \varepsilon_{r_{-} g} \varepsilon_{r_{-} a}$ are respectively relative electrical permittivity of water, ground, air, $c$ is velocity of e-m wave in vacuum, $\alpha$ is anisotropy factor, $\beta$ is ground structure factor. 

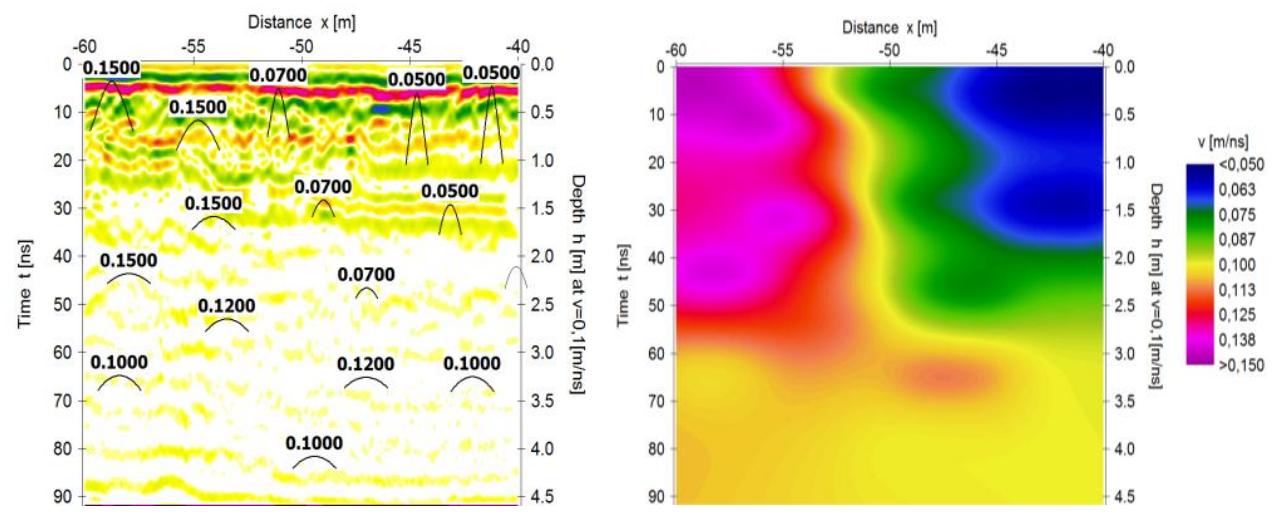

Fig. 9. A) Radargram with hypothetical diffraction hyperbolae; B) Velocity map obtained after application of DHA procedure.

Increasing of information or better resolution of radargram may be obtained by application of advanced procedures of signal processing. As an example, in Fig. 10 shown radargram recorded also in the Vistula river levee in Cracow but in other site than this presented in Fig. 1. In Fig. 10A, in zone A, direct waves were recorded and interpretation of anomalies was impossible. In zone B, stochastically distributed reflections were recorded which originate from loose zones existed in the levee; in this zone, two sub-zones B1 and B2 might be distinguished; the left sub-zone B1 has slightly higher amplitudes which may be interpreted as a more disintegrated medium than the right sub-zone B2; however, it is difficult to depict small regions with a higher leak risk in zone B. As shown in Fig. 10B application of more advanced processing allowed to outline with high accuracy the most dangerous small loose areas (violet colour) located especially in the sub-zone B1.

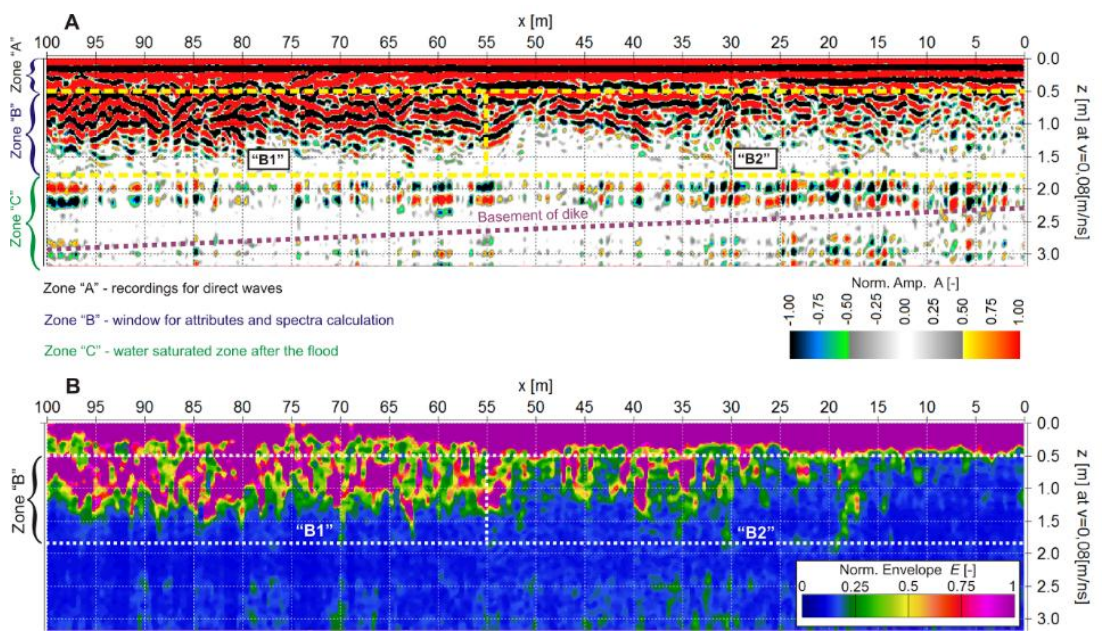

Fig. 10. A) radargram recorded on the Vistula river levee after basic (A) and advanced (B) signal processing [1].

Increasing of quality and resolution of radargrams may be also achieved by application of antennae with different constructions and using of modern electronic solutions of georadar systems. As an example, in Fig. 11 radargrams recorded with standard (classic) system (Fig. 11A) and modern HDR (High Dynamic Range) system (Fig. 11B) were presented. 
A

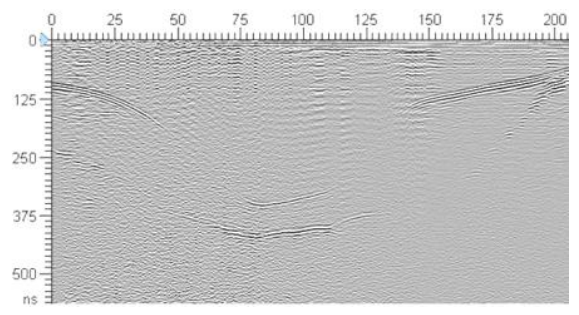

B

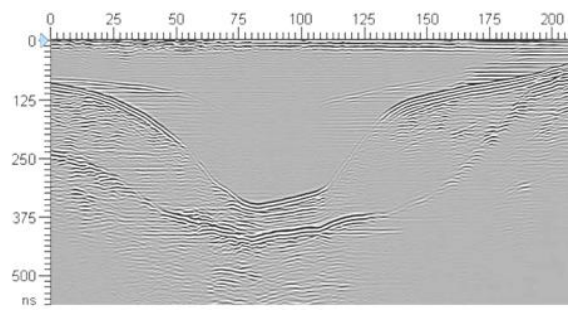

Fig. 11. A) radargrams recorded by using of standard (A) and modern (B) GPR systems (www.malagpr.com.au)

\section{Conclusions}

1. Short-offset reflection profiling (SORP) technique is valuable technique for noninvasive, continuous, fast and cheap examination of flood levees.

2. SORP should be used for interpolation of information delivered by punctual techniques of levee examination like boreholes, geotechnical soundings or measurements with sensors.

3. Disadvantage of SORP technique is fact that only qualitative interpretation may be performed. To improve quality and resolution of radargram, changeable orientation surveys should be carried out and recorded data have to be subjected to more advanced processing.

4. Procedure of Diffraction Hyperbola Analysis (if there is possibility) should be applied which allows to convert from qualitative to quantitative interpretation.

5. It obvious that application of modern solutions of georadar systems delivers better results of surveys than application of classic systems what was presented in the paper.

The works were financed by grant of Cracow University of Technology, no. Ś-2/335/2017/DS.

\section{References}

1. H. Marcak, T. Gołębiowski, The Use of GPR Attributes to Map a Weak Zone in a River Dike. Exploration Geophysics, 45(2), 125-133 (2014)

2. T. Gołębiowski, S. Tomecka-Suchoń, J. Farbisz, Application of Complex Geophysical Methods for Non-Invasive Examination of Technical Condition of River Dikes. Proc. of European Symp. on Modern Problems of Flood Protection, Paris-Orlean, , 233-241 (in Polish) (2012)

3. T. Gołębiowski, Application of the GPR Method for Detection and Monitoring of Objects with Stochastical Distribution in the Geological Medium. AGH Ed., p. 251 (in Polish) (2012)

4. T. Gołębiowski, Velocity Analysis in the GPR Method for Loose-Zones Detection in the River Embankments. Proceedings of International Conference "GPR 2010", Lecce, Italy (2010)

5. A. Kot, Expertise of Technical Condition of Flood Gate no. 1 Located in Left Flood Levee of the Vistula River in the Village of Wawrzeńczyce. Firm Aquin, Oświęcim (in Polish) (2013) 
6. J. Karczewski,Ł. Ortyl, M. Pasternak, Fundaments of GPR Method. AGH Ed., p. 346 (in Polish) (2012)

7. Mala, Basic and Advance Radar Theory. Documentation of MALA GeoScience, Sweden (2003)

8. E.D. Guy, J.J. Daniels, S. Radzevicius, Demonstration of Using Crossed Dipole GPR Antennae for Site Characterization. Geophysical Research Letters, 26(22), 3421-3424 (1999)

9. S. Radzevicius, Dipole antenna properties and their effects on ground penetrating radar data. Disertation. Bell\&Howell Information and Learning Company (2001)

10. M. Pasternak, Radar penetration of the ground, WKŁ Ed., (in Polish) (2014)

11. R. Mydlikowski, G. Beziuk, A. Szynkiewicz, Detection of inhomogeneities in structure of flood embankments by means of D.C. resistivity, gpr and frequency electromagnetic method measurements - short note, Acta Geodyn. Geomater., Vol. 4, No. 4 (148), 83$88,(2007)$

12. P. Popielski et al., The application of geophysical methods and numerical modeling for the evaluation of embankment technical conditions, Technical Transactions Environment Engineering, 1-ŚS/(2013) 